\title{
Determinantes de las innovaciones organizacionales: factores y obstáculos en la empresa española
}

\author{
M. Piedad Fernández Perea* \\ Universidad Nacional de Educación a Distancia
}

\section{Resumen}

Las innovaciones en las organizaciones (en adelante IO) llevan consigo la utilización de nuevos mecanismos organizativos de cara al funcionamiento interno de la empresa en áreas como la organización del lugar de trabajo o en las relaciones con el exterior a la empresa. Se han utilizado datos del Panel de Innovación Tecnológica (FECYT 2012). Se lleva a cabo un análisis de las variables que afectan a la adopción de IO en las compañías, realizando una estimación de un modelo estadístico probit trivariante, sobre el que se aplica el método de máxima verosimilitud. Los resultados evidencian relación de dependencia entre los diferentes mecanismos de innovación en las organizaciones y la necesidad de controlar esta interacción. Los resultados permiten evidenciar la existencia de nueve componentes o variables que tienen una relación positiva con la adopción de IO, entre ellas se encuentran la adquisición de I+D (I+D externa) y la necesidad de formación, corroborando que, para aquellas organizaciones que realizan un esfuerzo en incrementar su capacidad para adquirir nuevos conocimientos, la introducción de IO sirve como un complemento.

Palabras clave: innovaciones organizacionales; capacidades; recursos externos e internos; obstáculos.

Códigos JEL: O31.

\footnotetext{
" Autor de correspondencia. e-mail: piedad.fernandez.perea@gmail.com
}

ISSN 0212-1867 / e-ISSN 1989-3574

(C) ESIC Editorial, ESIC Business \& Marketing School

DOI: $10.7200 /$ esicm.162.0501.4e

http://www.esic.edu/esicmarket 


\section{Introducción}

Una IO es la implementación de nuevos métodos organizacionales en el negocio, en la organización del trabajo y/o en las relaciones con el exterior (Organización para la Cooperación y el Desarrollo Económicos, OCDE, 2015). La IO, como estrategia, es una salida clave para las empresas (Liao y Wu, 2010), una fuente de creación de valor (Hwang et al., 2008) y un elemento de difusión de diferentes prácticas organizativas (Armbruster et al., 2008). Las empresas participan en actividades de innovación por varias razones. Desde aumentar el rendimiento de la empresa mediante la reducción de los gastos administrativos o los costes de transacción, mejorar la satisfacción en el puesto de trabajo (y, por tanto, la productividad), el acceso a los bienes no transables entendidos como aquellos que son generados internamente y no pueden ser adquiridos por parte del entorno externo de la empresa o la reducción de los costes de suministro. Las organizaciones también adoptan IO como respuesta a cambios en el conocimiento gerencial y tecnológico, la competencia industrial, o expectativas futuras de directivos y accionistas sobre la mejora de su nivel de desempeño (Damanpour et al., 2009; Yamakawa y Ostos, 2012; Varela y Méndez, 2017).

La identificación de los determinantes que impulsan a las empresas a llevar a cabo innovaciones es útil cuando se examinan los factores que impulsan la actividad de innovación en la empresa. Además, gracias a la identificación de estos factores, la empresa puede elevar su competitividad y desarrollar su actividad en nuevos mercados (OCDE, 2015). En general, la inclusión de la innovación es un factor que facilita a la empresa una conducta de adaptación mediante cambios en la organización. Realizar actividades innovadoras permite a las organizaciones que introducen cambios tempranos ser conscientes de los últimos desarrollos, absorber conocimientos nuevos e incrementar sus oportunidades de beneficiarse de las actividades innovadoras (Cohen y Levinthal, 1990; Roberts y Amit, 2003).

Por tanto, se considera la relevancia, efectos, e importancia que para las empresas tiene la adopción y adaptación de IO. Sin embargo, y a pesar de aventurarse todos los beneficios que pudieran tener las IO, se hace necesario seguir profundizando en la dificultad que presenta el análisis de los objetivos de las IO. Por una parte, los problemas de conceptualización y, de otro la dificultad para su medición, ha dificultado la incorporación de un concepto operativo consensuado de IO. Ambas circunstancias han generado que gran parte de la literatura con relación a estos temas utilice conceptos distintos para referirse a estas innovaciones, con lo cual la posibilidad de establecer comparaciones y extender las conclusiones obtenidas en algunos estudios a un plano más general resulta complicado (Afcha, 2011).

La Oficina Europea de Estadística (EUROSTAT, 2005), en un intento por homogeneizar la medición y el análisis cuantitativo de las IO, integró a partir del 2001 al cuestionario de la Encuesta Europea de Innovación (CIS) cuestiones en relación con la incorporación de las IO y sus consecuencias en las organizaciones. Actualmente la definición de IO que es utilizada por el CIS se fundamenta en aquella que fue propuesta por el Manual de Oslo (OCDE, 2015); esto facilita llevar a cabo un 
seguimiento cuantificado de tres clases distintas de IO. Los datos utilizados a lo largo de este artículo corresponden al PITEC (FECYT, 2012).

La principal contribución de este artículo, con base en la información aportada anteriormente, consiste en determinar los factores que afectan a la integración de nuevos mecanismos organizativos en tres campos, i) la forma interna de trabajar en las organizaciones donde se incluyan mecanismos de business intelligence; ii) en la forma de organizar el lugar de trabajo, y iii) los nexos con el exterior que no habían sido usados previamente por la organización. Se trata de un asunto que hasta el momento no tiene un gran desarrollo literario y sobre el cual, a medida que aparezcan autores, permitirá incrementar el conocimiento acerca de los mecanismos que se relacionan con la incorporación o generación de IO.

Por otra parte, esta contribución se enmarca en la comprensión y entendimiento de los obstáculos o barreras que las empresas tienen cara a la adaptación y generación de $\mathrm{IO}$, como complemento al objetivo principal, ya que, a su vez, resultan determinantes a la hora de tomar la decisión de innovar en la parte organizacional de la empresa.

\section{Revisión de la literatura}

\subsection{La innovación organizacional}

Se considera que una IO incluye cualquier tipo de cambio que tenga como objetivo una optimización de los procesos integrados en el núcleo de la organización (OCDE, 2015). Dichos cambios llevan consigo la introducción de una idea o conducta que no existía previamente en la organización, y que la empresa incorpora con el objetivo de incrementar la rentabilidad (Daft, 1978; Damanpour y Evan, 1984; Birkinshaw et al., 2008).

En ocasiones, diferenciar entre procesos e innovaciones organizativas es un auténtico desafío, ya que ambos tipos de propuesta de innovación sirven para reducir costes a través de nuevas y más eficientes procesos de producción, distribución y organización interna. Es frecuente encontrarnos que muchas innovaciones van a contener aspectos de ambos tipos de innovación. Un punto de partida para el proceso de distinción en IO es el tipo de actividad: las innovaciones de proceso tratan principalmente acerca de la implementación de nuevos equipos, el software y las técnicas o procedimientos específicos, mientras que las IO se refieren principalmente a las personas y la organización del trabajo, siendo a menudo llamadas IO estructurales (EUROSTAT, 2005). Estas consisten en el cambio de responsabilidades, rendición de cuentas, líneas de decisión, y los flujos de información, así como el número de niveles jerárquicos de la estructura en la división de funciones y responsabilidades. En concreto, una IO es la implementación de un nuevo método organizativo en las prácticas empresariales de la empresa, la organización del lugar de trabajo o las relaciones exteriores (OCDE, 2015), que no ha sido utilizado antes en la empresa; todo ello es 
el resultado de las decisiones estratégicas adoptadas por la dirección. La literatura afirma que una IO es una salida importante para las empresas (Liao y Wu, 2010), una fuente de creación de valor (Hwang et al., 2008) y un indicador para la difusión interna dentro de las diferentes prácticas organizativas (Armbruster et al., 2008).

\subsection{Los objetivos de la innovación organizacional}

Una organización puede llevar a cabo actividades de innovación por diversas razones, que deben ser identificadas acorde a sus objetivos económicos (Guan et al., 2009). Dichos objetivos pueden estar relacionados con los productos, los mercados, la eficiencia, la calidad, o la capacidad de aprender y poner en práctica los cambios (EUROSTAT, 2005).

La percepción de la empresa por la sociedad, en lo que respecta a innovación y organización, sugiere que diferentes tipos de empresas pueden tener diferentes objetivos primarios de innovación (Leiponen y Helfat, 2009; Yang y Hsiao, 2009), debido a las variaciones en los patrones de innovación y el entorno en el que operan. Específicamente, Guan et al. (2009) muestran diferencias significativas acerca de la importancia de los objetivos de innovación basadas en su estado (empresas de alta tecnología frente a las no tecnológicas), la propiedad (las empresas estatales frente a las empresas no estatales), los recursos para la innovación (tiene departamento de $\mathrm{I}+\mathrm{D}$ versus ningún departamento de $\mathrm{I}+\mathrm{D}$ ), y el tamaño (las empresas pequeñas y medianas en comparación con las grandes empresas). La literatura también distingue entre innovación de producto e innovación de procesos. Leiponen y Helfat (2009) establecen que es probable que las empresas también tengan objetivos específicos para cada tipo de innovación. Las empresas no solo tienen que dirigir su atención a la mejora de la eficiencia y la productividad, sino también para desarrollar mecanismos de innovación encaminados a estimular la creación, el intercambio y la integración de conocimiento (Albers y Brewer, 2003). Esto significa que las actividades de innovación se conciben como un objetivo para mejorar el intercambio de conocimientos.

El mantenimiento, adquisición y evolución de las capacidades de una empresa dependen de sus objetivos de innovación y la estrategia de innovación resultante (Burgelman et al., 2005). Por tanto, los objetivos de innovación determinan las actividades y resultados de innovación.

En conclusión, podemos afirmar que las empresas buscan en la innovación una manera de obtener ventajas competitivas respecto a las demás empresas del sector a través de una reacción anticipada (Geroski, 1995; Roberts y Amit, 2003). La gran relevancia de las IO en la lucha empresarial se ha visto ratificada en diversos trabajos de investigación que tienen como objetivo un análisis del impacto de las IO sobre el desempeño o la rentabilidad de las empresas (Damanpour y Evan, 1984; Caroli y Van Reenen, 2001; Greenan, 2003; Piva et al., 2005). Todas estas investigaciones evidencian la existencia de dos puntos de vista diferente. Por un lado, nos señala que las IO funcionan como requisito previo de cara a la asimilación de cambio técnico 
$\mathrm{y}$, por tanto, tiene un efecto favorecedor de las innovaciones tecnológicas de proceso y producto. Por otro lado, encontramos una serie de autores que señalan que las IO son una gran fuente de ventajas competitivas, ya que por sí solas tienen un efecto relevante en la rentabilidad y productividad, así como en otros aspectos como pueden ser los plazos de entrega, calidad y/o flexibilidad (Womack et al., 1992; Hammer y Champy, 1993; Goldman et al., 1996).

El efecto de las IO sobre las empresas hace más fácil adquirir e integrar nuevo conocimiento funcionando estas innovaciones como un marco de referencia para generar innovaciones técnicas por parte de la organización. Debido a esto, llevar a cabo cambios dentro de las organizaciones se considera un requisito necesario para poder generar innovaciones tecnológicas (Lam, 2006). Dicho efecto se ha probado a través de diversos trabajos empíricos actuales, donde se observa que, si se llevan a cabo tanto innovaciones tecnológicas como organizacionales, son generadas sinergias que tienen un impacto sobre la productividad y desempeño de la organización (Damanpour et al., 2009; Polder et al., 2010; Yamakawa y Ostos, 2012; Varela y Méndez, 2017).

\subsection{Teoría de los recursos y capacidades en la adopción de innovaciones}

La primera referencia conocida, encargada de analizar la naturaleza y significatividad a nivel estratégico en la capacidad de diferenciarse por parte de una empresa, en nuevos productos y procesos fue Leonard-Barton (1992). Con posterioridad, diferentes autores (Henderson y Cockbum, 1994; De Carolis y Deeds, 1999; De Carolis, 2003; Sher y Yang, 2005) han llevado a cabo estudios, principalmente en el ámbito de altas tecnologías, sobre el resultado de las IO en el desempeño de los recursos y capacidades. También existen autores (Kusunoki et al., 1998; McEvily y Chakravarthy, 2002; Souitaris, 2002; Brusoni et al., 2005; Chen et al., 2006; Prajogo y Ahmed, 2006; Kleinschmidt et al., 2007; Fierro et al., 2013) que han intentado analizar la influencia que tienen factores como los recursos y las capacidades en el desempeño innovador de las organizaciones.

\subsubsection{Los recursos y las capacidades de las organizaciones}

Focalizar el análisis en los recursos y las capacidades de los que disponen las organizaciones parte de los dos fundamentos en los que se basa Barney (1991): 1) Las empresas se diferencian en gran medida en la capacidad y recursos de los que disponen o sobre los que mantienen cierto control, y 2) Esa diferencia mantiene una tendencia estable a lo largo del tiempo debido a que existen una serie de recursos que no se pueden adquirir en el mercado o que no pueden ser traspasados de una empresa a otra con la facilidad de otros.

La teoría que estableció Barney (1991) terminó derivando en dos enfoques diferentes; en primer lugar, se encuentra el enfoque basado en las capacidades dinámicas, 
entre los que destacan algunos autores (Teece et al., 1997; Eisenhardt y Martin, 2000). A medida que se ha necesitado llevar a cabo un análisis más profundo del mecanismo de creación o desarrollo de recursos y capacidades, y la actualización tanto de los recursos como de las capacidades que poseen las organizaciones en línea con los cambios que se producen en el entorno, estos aspectos han sido analizados en el enfoque basado en los recursos y capacidades de las organizaciones. El segundo enfoque que derivó de la teoría de Barney (1991) se basa principalmente en el conocimiento (Nonaka, 1994; Grant, 1996; Spender y Grant, 1996; Nonaka y Toyama, 2005), al haberse impuesto el rol de los recursos intangibles en la generación y mantenimiento de las ventajas competitivas (Barney, 1991; Grant, 1996).

Los recursos intangibles se basan principalmente en el conocimiento, el cual, al no ser cuantificable y medible, el codificarlo o cuantificarlo supone una ardua tarea que en muchos casos se convierte en imposible (Grant, 1999). Para Liebeskind (1996), el conocimiento es el activo más importante que puede tener una empresa. En esta investigación cobran una especial relevancia todos aquellos recursos basados en el conocimiento, como veremos más adelante en los resultados del modelo. La dificultad en lo referente al tratamiento del conocimiento se debe a que diversos autores han señalado el conocimiento como el principal elemento de los procesos de innovación (Miller y otros, 2007). Sin embargo, para que estos recursos tengan la capacidad de generar una ventaja competitiva que se mantenga a largo plazo, deben cumplir ciertas características: ser heterogéneos, imperfectamente imitables, sustituibles y móviles (Barney, 1991; Peteraf, 1993).

\subsubsection{Los recursos basados en conocimiento y el desempeño innovador}

En el ámbito de las actividades tanto tecnológica como no tecnológica, el conocimiento es uno de los principales orígenes de las ventajas competitivas (Kusunoki et al., 1998; McEvily y Chakravarthy, 2002). En multitud de situaciones la innovación tecnológica se debe a que se ha producido un incremento de conocimiento previo en la empresa. Este conocimiento ha sido desarrollado y acumulado de forma interna en la organización y transmitido a los individuos y grupos que han generado nuevos procesos o productos (Leonard-Barton, 1992).

Las empresas se enfrentan de diferentes maneras al reto de adquirir nuevo conocimiento. La manera de adquirir conocimiento se fija desde la parte estratégica de la empresa. Esto tiene ciertos efectos para los procesos de innovación sin importar del tipo que estos sean. Si nos enfocamos en el conocimiento tecnológico, este se puede incrementar bien desarrollándolo de forma interna en la organización o adquiriéndolo a través de relaciones que la organización pueda mantener con otras entidades del exterior (Sher y Yang, 2005; Fierro et al., 2013). Por todo ello se ha de distinguir entre aquellos recursos que se fundamentan en un conocimiento generado de manera interna de la empresa (conocimiento endógeno) y aquel que se basa principalmente en la adquisición e integración de conocimiento del exterior (conocimiento exógeno); la elección de uno u otro dependerá de factores tales como el tamaño de la empresa 
o el sector. Asimismo existe una extensa literatura acerca de la gestión del conocimiento que se centra en diferenciar entre lo que se denomina conocimiento tácito y el conocimiento explícito (Nonaka, 1994; Evans et al., 2002; Holden y Glisby, 2010). El conocimiento explícito tiene mayor facilidad para ser explotado por la empresa, pero, dado que se caracteriza por una gran facilidad para ser transferido, es también más fácil de ser imitado por otras empresas. Por otro lado, el conocimiento tácito es el caso contrario, y esta dificultad para ser transferido es lo que le permite que sea con mayor probabilidad una ventaja competitiva sostenible. Por tanto, los recursos pueden tener una incidencia significativa en su carácter innovador en función de su grado de codificación (McEvily y Chakravarthy, 2002; Edmondson et al., 2003; Brusoni et al., 2005). Edmondson et al. (2003) apuntan que habrá más heterogeneidad entre el desempeño de las empresas cuando este se basa en conocimiento tácito que cuando se basa en conocimiento explícito. Por ello, se considera básico distinguir entre recursos basados en conocimiento tácito y recursos basados en conocimiento explícito.

El conocimiento tácito es fundamentalmente subjetivo, encontrándose muy arraigado en las organizaciones; este se basa en la experiencia, las creencias y las perspectivas de los individuos (Schulz y Jobe, 2001). Este conocimiento es generado y transferido a través de las interrelaciones que se llevan a cabo entre los distintos individuos que forman la organización. Es por ello que una parte del conocimiento necesitado para ser capaces de innovar se encuentra en las organizaciones y es utilizado por los individuos (Subramaniam y Youndt, 2005).

Pese a que en la actualidad el conocimiento tácito es considerado clave de cara a tener capacidad para generar una ventaja competitiva a largo plazo, este no puede suplir totalmente el conocimiento explícito (codificado). Balconi (2002) señala que el conocimiento tácito debe apoyarse en el codificado, tal y como señalan Brusoni et al. (2005), quienes declaran que las inversiones que las organizaciones llevan a cabo en $\mathrm{I}+\mathrm{D}$ y en el personal de I+D ayudan a acceder a fuentes de conocimiento codificado.

Por lo general, un gran porcentaje de empresas no disponen de los recursos necesarios que les permita conseguir un alto nivel de productividad de sus procesos de innovación tecnológica y no tecnológica. Es por ello por lo que dichas organizaciones deben tener en cuenta a la hora de innovar un producto, servicio o proceso, que no solo deben poseer un conocimiento endógeno. Sino que estas organizaciones deben tener la capacidad de identificar e integrar aquel conocimiento exógeno que sea determinante para llevar a cabo sus procesos de innovación (De Propris, 2000). Sin embargo, es arduo para una organización la tarea de llevar a cabo una evaluación, absorción y utilización de un conocimiento exógeno si la organización no tiene las habilidades necesarias para generarlo de manera interna (Cohen y Levinthal, 1990). Ambos autores señalan un concepto nuevo, la capacidad de absorción, con el que designan a la habilidad para valorar adecuadamente una nueva información, asimilarla y utilizarla con fines comerciales. Henderson y Cockbum (1994) basan sus argumentos en que la habilidad de la organización para conseguir e integrar ese conocimiento de origen externo es una destacable ventaja competitiva. Dicha 
habilidad tan solo puede adquirirse a través del denominado learning by doing (Cohen y Levinthal, 1990). Este conocimiento adquirido del exterior está compuesto tanto por una parte de conocimiento tácito como explícito. En lo que se refiere al primero, este tiene dificultad para ser traspasado entre organizaciones si no existe entre estas un vínculo estrecho.

\subsection{Los obstáculos o barreras a la innovación}

Hay gran cantidad de estudios que hablan acerca de cómo las barreras a las que se enfrenta la innovación dependen de unos factores concretos. Uno de los más importantes es el del tamaño (Piatier, 1984; Dougherty, 1992; Henderson, 1993): donde las organizaciones de pequeño tamaño y de reciente creación se encuentran con más obstáculos (Hadjimanolis, 2003; MacCann, 2009). Otro factor clave es la fase en la que se encuentre el grado de innovación, en el momento en el que se produce la decisión de innovar o una vez que esta ha comenzado su proceso innovador, al incrementar sus esfuerzos (Mohnen y Röller, 2005).

La ubicación de las organizaciones es otro punto a tener en cuenta a la hora de innovar, ya que las legislaciones, infraestructuras, regulaciones... no son iguales en todos los lugares (Tourigny y Le, 2004). También determinan que el sector es otro factor de importancia. En el estudio de estos autores puede verse cómo las empresas con sectores de baja y media intensidad tecnológica se enfrentan a menos barreras que los que son altamente tecnológicos.

\section{Innovaciones organizacionales: un modelo estadístico}

Los datos utilizados corresponden al PITEC 2012. Esta encuesta, aplicada con carácter anual desde 2001, replica para España el cuestionario utilizado por el CIS. De esta forma, las preguntas utilizadas en el PITEC coinciden con las distintas oleadas del CIS.

El cuestionario PITEC define las IO como la implementación de nuevos métodos organizativos en tres ámbitos: i) el funcionamiento interno de la empresa (incluyendo métodos/sistemas de gestión del conocimiento); ii) en la organización del lugar de trabajo, y iii) en las relaciones externas que no han sido utilizadas previamente por la empresa. Dicha implementación debe ser el resultado de decisiones estratégicas llevadas a cabo por la dirección de la empresa. Excluye fusiones y adquisiciones, aunque estas supongan una novedad organizativa para la empresa.

Una IO debe ser el resultado de decisiones estratégicas llevadas a cabo por la dirección de la empresa. La decisión respecto al tipo de IO emprendida no parece una decisión independiente, sino, por el contrario, una decisión que se realiza de manera simultánea. La aplicación de modelos univariantes para determinar la probabilidad de introducir una innovación organizacional de alguno de los tres tipos 
señalados anteriormente no sería adecuada para el análisis de este tipo de situaciones, dado que podría estar correlacionada.

Para solventar este problema se propone la estimación de un modelo probit trivariante, modelo de regresión que permite la estimación simultánea de tres variables dependientes dicotómicas. Este modelo contempla de forma explícita la existencia de autocorrelación en el término de error y, por tanto, permite controlar el sesgo ocasionado por la existencia de correlación entre variables no observables que determinan la adopción de las distintas innovaciones.

El modelo probit trivariante que permite identificar las variables que influyen sobre la introducción de IO está determinado por las variables descritas en la tabla 1:

Tabla 1. Descripción de las variables de IO

\begin{tabular}{ll}
\hline \multicolumn{1}{c}{ Variables dependientes } & \multicolumn{1}{c}{ Descripción } \\
\hline Innovación organizacional 1 $\left(\mathrm{IO}_{1}\right)$ & $\begin{array}{l}\text { Nuevas prácticas empresariales en la organización del } \\
\text { trabajo o de los procedimientos de la empresa. }\end{array}$ \\
\hline Innovación organizacional 2 $\left(\mathrm{IO}_{2}\right)$ & $\begin{array}{l}\text { Nuevos métodos de organización de los lugares de trabajo } \\
\text { en su empresa con el objetivo de un mejor reparto de } \\
\text { responsabilidades y toma de decisiones. }\end{array}$ \\
\hline Innovación organizacional 3 $\left(\mathrm{IO}_{3}\right)$ & $\begin{array}{l}\text { Nuevos métodos de gestión de las relaciones externas con } \\
\text { otras empresas o instituciones públicas. }\end{array}$ \\
\hline
\end{tabular}

Fuente: Elaboración propia.

$$
\begin{aligned}
& I O_{1}^{*}=\beta_{11} x+\varepsilon_{1} I O=1 \text { si } I O_{1}^{*}>0, \\
& I O_{2}^{*}=\beta_{21} x+\varepsilon_{2} I O=1 \text { si IO }{ }_{2}^{*}>0, \\
& I O_{3}^{*}=\beta_{31} x+\varepsilon_{31} I O=1 \text { si IO } I_{3}^{*}>0,
\end{aligned}
$$

Donde $I O_{1}^{*}, I O_{2}^{*}$ y $I O_{3}^{*}$ representan los verdaderos valores no observados de la propensión a introducir IO relacionadas con: i) la introducción de nuevas prácticas empresariales en la organización del trabajo o de los procedimientos de la empresa (incluye métodos/sistemas de gestión del conocimiento); ii) nuevos métodos de organización de los lugares de trabajo en su empresa con el objetivo de un mejor reparto de responsabilidades y toma de decisiones, y iii) nuevos métodos de gestión de las relaciones externas con otras empresas o instituciones públicas. $I O_{1}, I O_{2}$ y $I O_{3}$ son las variables dicotómicas observadas que indican si la empresa introdujo alguno de los tipos de IO mencionados anteriormente; $\mathrm{X}$ es un vector de variables que influyen sobre la probabilidad de introducir algún tipo de innovación, y $\varepsilon$, el término de error aleatorio.

A partir de la estimación de este modelo probit es posible calcular las predicciones de la probabilidad de introducir una IO tipo 1, 2 o 3, e incorporarla para determinar el efecto de las IO. El resultado de esta primera estimación permitirá, por un lado, cumplir con el primer objetivo planteado de identificar los determinantes 
de los tres tipos de innovación organizacional y, por otro lado, resolver el problema de selección muestral.

En el periodo de 2010 a 2012, objeto de estudio, usaremos el 100\% de las empresas que conforman la muestra del PITEC (véase tabla 2), esto es, 9.612 empresas que han llevado a cabo actividades de innovación tecnológica y no tecnológica.

Tabla 2. Distribución del número de empresas innovadoras según clasificación PITEC (2012)

\begin{tabular}{lccc}
\hline & $\begin{array}{c}\text { Número } \\
\text { de empresas }\end{array}$ & $\begin{array}{c}\text { Empresas } \\
(\%)\end{array}$ & $\begin{array}{c}\text { Porcentaje } \\
\text { acumulado }\end{array}$ \\
\hline 1. MEG & 1.584 & 19,3 & 19,3 \\
2. MID & 5.765 & 60,0 & 79,3 \\
3. MFG+MID & 961 & 10,0 & 89,3 \\
4. MIDE & 329 & 3,4 & 92,7 \\
5. MEP & 703 & 7,3 & 100,0 \\
\hline Total & 9.612 & 100,0 & \\
\hline
\end{tabular}

Fuente: Elaboración propia.

\subsection{Medida y descripción de las variables independientes que influyen en la adopción de IO}

Como ya se ha indicado en el apartado anterior, se utiliza la información del PITEC para obtener las proxies de cada una de las variables implicadas. Vemos en la tabla 3 una descripción de las variables independientes que afectan en la adopción de IO y que han sido utilizadas en nuestro modelo para ver el efecto que sobre las IO tienen estas en las empresas.

En apartados anteriores hemos ido identificando aquellas variables que están más directamente vinculadas a la teoría de los recursos y capacidades de la empresa, y más en concreto en las teorías sobre el tipo de conocimiento que la empresa necesita para poder llevar a cabo actividades de innovación. En nuestro modelo hemos incorporado otras variables que hemos entendido pueden cumplir con el objetivo planteado en este artículo; qué factores, según el enfoque utilizado, influyen en las actividades de innovación en relación con la incorporación de IO en la empresa (véase tabla 4).

A continuación se enumeran las variables independientes utilizadas para estimar la regresión que identifica los factores que afectan la adopción de los diferentes tipos de IO. La tabla 4 muestra estas variables clasificadas en torno a cuatro dimensiones. En primer lugar se incluye un conjunto de variables que indican las actividades innovadoras llevadas a cabo por la empresa para conseguir nuevos productos, servicios o procesos, basados en la aplicación de ciencia y tecnología. 
Tabla 3. Variables independientes que afectan la adopción de IO

\begin{tabular}{|c|c|}
\hline Factores tecnológicos & $\begin{array}{l}\text { Adquisición de I+D (I+D externa) } \\
\text { Adquisición de maquinaria, equipo y software. } \\
\text { Adquisición de otros conocimientos externos de innovación } \\
\quad \text { (no incluido en I+D) } \\
\text { Introducción de innovaciones en el mercado } \\
\text { Diseños y otros preparativos } \\
\text { Solicitud de patentes } \\
\text { Uso protección: registro de modelos de utilidad }\end{array}$ \\
\hline Obstáculos a la innovación & $\begin{array}{l}\text { Falta de fondos de la empresa } \\
\text { Falta de financiación por fuentes externas } \\
\text { Costes de innovación elevados } \\
\text { Falta de personal cualificado } \\
\text { Falta de información sobre tecnología } \\
\text { Falta de información sobre mercados } \\
\text { Dificultad para encontrar socios } \\
\text { No es necesario porque no hay demanda de innovaciones }\end{array}$ \\
\hline Recursos humanos & $\begin{array}{l}\text { Número de empleados en } t \\
\text { Formación } \\
\text { Número de trabajadores en I+D interna } \\
\text { Número de consultores externos }\end{array}$ \\
\hline Financiación & $\begin{array}{l}\text { F. Pública: administraciones locales o autonómicas } \\
\text { F. Pública: Administración del Estado } \\
\text { F. Pública: Unión Europea }\end{array}$ \\
\hline
\end{tabular}

Fuente: Elaboración propia a partir de PITEC (2012).

Como se ha remarcado, las IO permiten potenciar otras innovaciones de la empresa, con lo cual las IO pueden ser también concebidas como una vía para superar parte de los obstáculos a los que se enfrentan las empresas para poder innovar. Es por ello que se han incorporado las variables que indican si la empresa presenta determinadas barreras para innovar siguiendo las preguntadas en la EIN ${ }^{1}$.

El tamaño de la empresa, medido por el número de empleados, ha sido señalado en trabajos empíricos como un factor determinante en la adopción de IO. Se espera que las empresas de mayor tamaño tengan los recursos necesarios para emprender IO y que, por otro lado, la necesidad por mantener una estructura organizacional adecuada a sus dimensiones genera una presión adicional en las empresas de mayor dimensión. Asimismo, la incorporación de IO requiere fundamentalmente de trabajadores cualificados para emprender cambios a nivel interno. Para medir la influencia de este factor se tiene en cuenta el número de empleados dedicados a actividades de I+D según su titulación.

(1) Factores de coste: falta de fondos internos, externos, y coste elevado. Factores de conocimiento: falta de personal cualificado, falta de información sobre tecnología, falta de información sobre los mercados y dificultad para encontrar socios. Factores de mercado: dominio de empresas establecidas, incertidumbre respecto a la demanda. Otros motivos para no innovar: falta de demanda de innovaciones y no necesidades de innovación en los mercados. 
Por último, se incluyen tres variables relacionadas con la capacidad financiera de la empresa para emprender IO. Para ello, se utiliza el porcentaje de fondos públicos recibidos para emprender actividades de carácter innovador.

Tabla 4. Descripción de las variables independientes según el enfoque utilizado

\begin{tabular}{|c|c|c|}
\hline Enfoque & Variables & Medida de las variables \\
\hline $\begin{array}{l}\text { Recursos basados en } \\
\text { conocimiento endógeno } \\
\text { tácito }\end{array}$ & $\begin{array}{l}\text { Número de trabajadores en } \\
\text { I+D interna }\end{array}$ & $\begin{array}{l}\text { Número de trabajadores dedicados } \\
\text { a actividades de } \mathrm{I}+\mathrm{D} \text { interna: proxy }\end{array}$ \\
\hline \multirow{2}{*}{$\begin{array}{l}\text { Recursos basados en } \\
\text { conocimiento endógeno } \\
\text { explícito }\end{array}$} & \multirow{2}{*}{$\begin{array}{l}\text { Los derechos de propiedad } \\
\text { industrial }\end{array}$} & $\begin{array}{l}\text { Solicitud de patentes (innovaciones } \\
\text { significativas o radicales): dummy }\end{array}$ \\
\hline & & $\begin{array}{l}\text { Uso de protección: registro de } \\
\text { modelos de utilidad (innovaciones } \\
\text { incrementales): dummy }\end{array}$ \\
\hline \multirow{3}{*}{$\begin{array}{l}\text { Recursos basados en } \\
\text { conocimiento exógeno } \\
\text { tácito }\end{array}$} & \multirow{3}{*}{$\begin{array}{l}\text { Adquisición de conocimiento } \\
\text { externo }\end{array}$} & $\begin{array}{l}\text { Adquisición de otros } \\
\text { conocimientos externos de } \\
\text { innovación (no incluido en } \mathrm{I}+\mathrm{D} \text { ): } \\
\text { dummy }\end{array}$ \\
\hline & & Gastos en formación para I+D \\
\hline & & $\begin{array}{l}\text { Número de consultores externos: } \\
\text { dummy }\end{array}$ \\
\hline \multirow{2}{*}{$\begin{array}{l}\text { Recursos basados en } \\
\text { conocimiento exógeno } \\
\text { explícito }\end{array}$} & $\begin{array}{l}\text { Adquisición de conocimiento } \\
\text { externo }\end{array}$ & $\begin{array}{l}\text { Adquisición de I+D (I+D externa): } \\
\text { dummy }\end{array}$ \\
\hline & Inversión en I+D & $\begin{array}{l}\text { Adquisición de maquinaria, equipo } \\
\text { y software: dummy }\end{array}$ \\
\hline
\end{tabular}

Fuente: Elaboración propia.

\section{Innovaciones organizacionales: resultados}

Los resultados de la estimación probit trivariante se muestran en la tabla 5, “determinantes de la introducción de IO". Cabe destacar, como ya se ha anticipado en la descripción del modelo, la significancia estadística de los coeficientes de correlación (véase tabla 6) entre los distintos tipos de IO. Este resultado confirma la necesidad de adoptar una aproximación multivariante para obtener una correcta predicción de la probabilidad de introducir IO. Dada la naturaleza de los tipos de IO, en la que la existencia de elementos comunes en las empresas que adoptan alguno o varios de los distintos tipos de IO podría ser una explicación a esta interdependencia.

Siguiendo este planteamiento, los resultados sitúan la capacidad de adquisición de conocimiento como un elemento que actúa de forma transversal en la adopción de distintas IO (Chang y Lee, 2008). En el presente caso de estudio, los resultados 
obtenidos permiten identificar nueve variables que afectan positivamente la adopción de los tres tipos de IO; se trata de las variables relacionadas con la "Adquisición de $\mathrm{I}+\mathrm{D}$ (I+D externa)", "Adquisición de maquinaria, equipo y software", "Introducción de innovaciones en el mercado" y "Uso de protección: registro de modelos de utilidad", en lo que respecta a los factores tecnológicos; en cuanto a los obstáculos a la innovación, nos encontramos con que el factor de "No necesitadas las innovaciones por falta de demanda de innovaciones" también ha tenido una relación positiva en los tres tipos de IO; por el lado de los recursos humanos, vemos que el "Número de empleados en el periodo $t$ ", "Actividades formativas de los empleados" en el periodo corriente y "Número de consultores externos" también tienen un efecto positivo, encontrándonos por último la "Financiación de las administraciones públicas", la cual también muestra una relación positiva. Estas variables afectan claramente la capacidad de adquisición de conocimiento de la empresa, confirmando, en cierta forma, que en aquellas empresas que refuerzan su capacidad de asimilación de nuevo conocimiento las introducciones de IO tienen un carácter complementario, es decir, la adopción de una de ellas influye positivamente en la propensión a adoptar otro tipo distinto de innovación organizacional.

Los autores Damanpour et al. (2009) y Polder et al. (2010) muestran una similar relación cuando analizan la interacción entre innovaciones no tecnológicas e innovaciones tecnológicas, confirmando que, en presencia de IO, la introducción de innovaciones de carácter tecnológico tiene un mayor efecto sobre el desempeño de la empresa.

A continuación se analizarán las relaciones obtenidas en cuanto a cada uno de los tres tipos de IO analizadas (tabla 5). Las tres primeras columnas muestran los resultados respecto a la introducción de nuevas prácticas empresariales en la organización del trabajo o de los procedimientos de la empresa, $\mathrm{IO}_{1}$; por tanto, todo lo concerniente a la gestión del conocimiento. Este tipo de innovaciones se ven positivamente afectadas por aquellos factores relacionados con los factores tecnológicos y con los flujos de información de la empresa. Esto queda parcialmente confirmado por la influencia positiva que ejerce el tamaño de la empresa sobre la introducción de nuevos sistemas de conocimiento, ya que, por lo general, las empresas de mayor dimensión manejan flujos de información de mayor magnitud, aumentando con ello la complejidad en su gestión y correcta asimilación. Otro elemento que influye significativamente en la adopción de nuevos sistemas de gestión del conocimiento es la introducción de tecnologías de la información y de la comunicación (TIC). La significancia estadística de la variable de adquisición de maquinaria, software y hardware confirma la importancia de estas tecnologías en la implementación de IO. La relevancia de las TIC en la implementación de IO ha quedado patente en los trabajos de Polder et al. (2010) y Gu y Gera (2004). También influyen significativamente la introducción de innovaciones en el mercado, así como la introducción de preparativos para productos y distribución. Por último, como factores tecnológicos, debemos considerar la influencia positiva de las solicitudes de patentes y el uso de protección: registro de modelos de utilidad. 
Tabla 5. Determinantes de la introducción de IO. Modelo probit trivariante

\begin{tabular}{|c|c|c|c|c|c|c|c|c|c|}
\hline & \multicolumn{3}{|c|}{ Innov. org. 1} & \multicolumn{3}{|c|}{ Innov. org. 2} & \multicolumn{3}{|c|}{ Innov. org. 3} \\
\hline & Coef. & Error & Sig. & Coef. & Error & Sig. & Coef. & Error & Sig. \\
\hline \multicolumn{10}{|l|}{ Factores tecnológicos } \\
\hline Aquisición de I+D (I+D externa) & ,173 & ,029 &, $000^{* * * *}$ & ,167 & ,029 &, $000^{* * * *}$ & ,180 & ,036 &, $000^{* \ldots *}$ \\
\hline $\begin{array}{l}\text { Adquisición de máquinas, } \\
\text { equipo y sotfware }\end{array}$ &, 237 &, 034 &, $000^{* * * *}$ & ,156 &, 035 &, $000^{* * * *}$ & ,095 &, 043 &, $027^{* * *}$ \\
\hline $\begin{array}{l}\text { Introducción de innovaciones } \\
\text { en el mercado }\end{array}$ & ,181 &, 032 &, $000^{* * * *}$ & ,186 &, 032 &, $000^{* * *}$ & ,233 &, 038 &, $000^{* * * *}$ \\
\hline $\begin{array}{l}\text { Adquisición de conocimientos } \\
\text { externos }\end{array}$ &,- 062 & ,087 & ,480 &,- 001 & ,087 & ,995 &,- 025 & ,103 &, 805 \\
\hline $\begin{array}{l}\text { Introducción de preparativos } \\
\text { para producción/distribución }\end{array}$ & ,109 &, 048 &, $024^{* *}$ & ,071 & ,049 & ,147 & ,009 & ,059 &, 874 \\
\hline Solicitud de patentes & ,093 &, 038 &, $015^{* * * *}$ & ,091 & ,039 &, $018^{* * *}$ &, 070 &, 046 & ,129 \\
\hline $\begin{array}{l}\text { Uso de protección: registro } \\
\text { de modelos de utilidad }\end{array}$ & ,164 &, 049 &, $001^{* * * *}$ & ,160 & ,049 &, $001^{* * * *}$ & ,141 & 059 &, $017^{* *}$ \\
\hline \multicolumn{10}{|l|}{ Obstáculos a la innovación } \\
\hline $\begin{array}{l}\text { Falta de fondos dentro de } \\
\text { la empresa o grupo }\end{array}$ & ,009 &, 016 &, 571 &, 012 & ,016 &, 460 &, 021 &, 021 & ,328 \\
\hline $\begin{array}{l}\text { Falta de financiación externa } \\
\text { a la empresa }\end{array}$ &,- 013 & ,016 & ,424 &,- 019 &, 016 & ,242 &,- 049 &, 021 &, $023^{* * *}$ \\
\hline Costes de innovación elevados &,- 006 &, 014 & ,651 &,- 002 &, 014 & ,903 & 019 &, 018 & ,289 \\
\hline Falta de personal cualificado &,- 032 &, 018 &, $076^{*}$ &,- 031 & ,018 & ,095* & ,020 &, 024 & ,389 \\
\hline $\begin{array}{l}\text { Falta de información sobre } \\
\text { tecnología }\end{array}$ &,- 028 & ,023 & ,228 & ,003 &, 024 & ,896 & ,036 &, 030 & ,235 \\
\hline $\begin{array}{l}\text { Falta de información sobre los } \\
\text { mercados }\end{array}$ &,- 012 &, 020 &, 541 &,- 026 &, 020 & ,196 &,- 073 & ,026 &, $005^{* * *}$ \\
\hline $\begin{array}{l}\text { Dificultad en encontrar socios } \\
\text { para la coop. en innovación }\end{array}$ &, 000 &, 014 & ,979 &,- 007 & ,014 & ,639 &,- 076 & ,018 &, $000^{* * * *}$ \\
\hline $\begin{array}{l}\text { No necesitadas falta de demanda } \\
\text { de innovaciones }\end{array}$ & ,126 & ,012 &, $000^{* * * *}$ & ,124 & ,012 &, $000^{* * * *}$ &, 111 & ,016 &, $000^{* * *}$ \\
\hline \multicolumn{10}{|l|}{ Recursos humanos } \\
\hline Número de empleados en $t$ & 000 &, 000 &, $000^{* * * *}$ &, 000 &, 000 &, $000^{* * * *}$ &, 000 &, 000 &, $000^{* * * *}$ \\
\hline Gastos en formación para I+D &, 328 &, 035 &, $000^{* * * *}$ & ,320 &, 035 &, $000^{* * * *}$ & ,278 & ,042 &, $000^{* * *}$ \\
\hline $\begin{array}{l}\text { Número de trabajadores } \\
\text { en I+D interna }\end{array}$ &, 000 &, 000 & ,252 &, 000 &, 000 & ,191* &, 001 &, 000 &, $002^{* * * *}$ \\
\hline No consultores externos & ,128 & 056 &, $022^{* * *}$ & 236 & ,055 &, $000^{* * *}$ & ,162 & ,065 &, $013^{* *}$ \\
\hline
\end{tabular}


Determinantes de las innovaciones organizacionales: factores y obstáculos...

\begin{tabular}{lcccccccccc}
\hline & \multicolumn{3}{c}{ Innov. org. 1} & \multicolumn{3}{c}{ Innov. org. 2 } & \multicolumn{3}{c}{ Innov. org. 3 } \\
\hline & Coef. & Error & Sig. & Coef. & Error & Sig. & Coef. & Error & Sig. \\
\hline Financiación & & & & & & & & & \\
\hline $\begin{array}{l}\text { Financ. pública: administraciones } \\
\text { locales o autonómicas }\end{array}$ &, 079 &, 034 &, $020^{* *}$ &, 024 &, 035 &, 494 &, 044 &, 042 &, 292 \\
$\begin{array}{l}\text { Financ. pública: Administración } \\
\text { del Estado }\end{array}$ &, 075 &, 033 &, $023^{* *}$ &, 082 &, 033 &, $013^{* * *}$ &, 169 &, 039 &, $000^{* * * *}$ \\
$\begin{array}{l}\text { Financ. pública: Unión Europea } \\
\text { Intersección }\end{array}$ & 096 &, 050 &, $054^{*}$ &, 041 &, 051 &, 419 &, 120 &, 058 &, $038^{* * *}$ \\
\hline & $-1,457$ &, 055 &, $000^{* * *}$ & $-1,482$ &, 055 &, $000^{* * * *}$ & $-1,865$ &, 073 &, $000^{* * * *}$ \\
\hline
\end{tabular}

**** Significativo al 99\% de confianza.

** Significativo al $95 \%$ de confianza.

* Significativo al $90 \%$ de confianza.

Fuente: Elaboración propia a partir de PITEC (2012).

En cuanto a los obstáculos o barreras a la innovación, vemos que tiene una influencia muy significativa la variable que hace referencia a no llevar a cabo innovaciones debido a no ser necesitadas por falta de demanda del mercado. Encontrando la variable "falta de personal cualificado" algo significativa.

Las variables relacionadas con la cualificación de los recursos humanos también muestran una influencia significativa sobre la incorporación de este tipo de IO. Tanto la inversión en formación como el reconocimiento por parte de la empresa de falta de personal cualificado para desarrollar adecuadamente sus innovaciones muestran que las empresas que incorporan nuevos sistemas de gestión del conocimiento están interesadas en mejorar y superar las barreras originadas por el déficit en capital humano revelado por la empresa. Asimismo, se muestra como positivamente significativa la necesidad de contar con consultores externos expertos que trabajan in situ en la empresa.

Por último, observamos las variables relacionadas con la financiación pública de las actividades de innovación, donde se observa una influencia positiva de los tres tipos de financiación propuesta, siendo la financiación por parte de la Unión Europea la menos significativa.

Tabla 6. Correlaciones entre los tres tipos de IO

\begin{tabular}{lcl}
\hline \multicolumn{3}{c}{ Correlaciones (rho de Spearman) } \\
\hline & Coef. & Sig. \\
\hline Inn. org. 2 - Inn. org. 1 &, $708^{* * *}$ & 0,000 \\
Inn. org. 3 - Inn. org. 1 &, $473^{* * *}$ & 0,000 \\
Inn. org. 2 - Inn. org. 3 &, $488^{* * *}$ & 0,000 \\
\hline
\end{tabular}

"* La correlación es significativa al nivel 0,01 (bilateral).

Fuente: Elaboración propia. 
Las columnas 3, 4 y 5 pertenecen a la estimación sobre las IO que implican nuevos métodos de organización en los lugares de trabajo, $\mathrm{IO}_{2}$. Estas innovaciones están asociadas a la mejora en el reparto de responsabilidades, el proceso de toma de decisiones o la gestión de sus equipos de trabajo. En este sentido, los resultados muestran que las empresas que invierten en la formación de sus trabajadores, en la adquisición de equipos que puedan conducir a una mayor productividad de dichos trabajadores (inversión en maquinaria, hardware y software) y en la contratación de consultores externos, tienen una mayor probabilidad de adoptar nuevos métodos para la organización de los lugares de trabajo.

Dentro de los factores tecnológicos, al igual que ocurría con la $\mathrm{IO}_{1}$, la variable "introducción de innovaciones en el mercado" ofrece una relación positiva, así como el uso de protección: registro de modelos de utilidad, siendo la variable "solicitud de patentes" algo menos significativa.

Al igual que ocurría con la $\mathrm{IO}_{1}$, en lo referente a los obstáculos o barreras a la innovación, vemos que tiene una influencia muy significativa la variable que hace referencia a no llevar a cabo innovaciones debido a no ser necesitadas por falta de demanda del mercado. Encontrando la variable "falta de personal cualificado" algo significativa.

Nuevamente el tamaño de la empresa influye positivamente sobre la posibilidad de introducir una innovación de este tipo. Esto responde a una mayor necesidad por parte de las empresas de mayor dimensión por mantener un nivel de organización laboral y una estructura organizacional adecuada al tamaño de la empresa. Este resultado es confirmado por Fariñas y López (2011), quienes encuentran que la propensión a introducir IO del tipo 1, 2 y 3 es creciente con el tamaño de la empresa.

En lo que respecta a las variables relacionadas con la financiación pública de las actividades de innovación, observamos que únicamente la financiación pública de la Administración del Estado ofrece una relación positiva significativa.

En cuanto a las $\mathrm{IO}$ del tipo $\mathrm{IO}_{3}$, mejora en los métodos de gestión de las relaciones externas, influye, tal y como cabía esperar, que las empresas realicen actividades externas de I+D; esto es debido a principalmente a que las empresas muestran una mayor capacidad para mejorar los índices de productividad, así como una menor incertidumbre sobre los resultados y sobre los beneficios de la misma. Al igual que en los casos de las $\mathrm{IO}_{1}$ e $\mathrm{IO}_{2}$, la formación de recursos humanos y la contratación de consultores externos se revelan como factores determinantes para conseguir mejoras en la gestión de las relaciones externas de la empresa. Otras variables relacionadas con los factores tecnológicos como son la introducción de innovaciones en el mercado y el uso de protección: registro de modelos de utilidad, muestran una relación positiva significativa que facilita igualmente que se lleven a cabo nuevos métodos de gestión para las relaciones externas de la empresa.

En cuanto a las variables que hacen referencia a los obstáculos o barreras a la innovación, vemos que hay diferencias con respecto a las anteriores $\mathrm{IO}_{1}$ e $\mathrm{IO}_{2}$, ya que observamos que la falta de información sobre los mercados, la dificultad en encontrar socios para la cooperación en innovación, o el considerar no llevar a cabo 
innovaciones por no estar necesitadas debido a la falta de demanda, son factores determinantes para llevar a cabo este tipo de IO; constatamos que los costes elevados de innovación también es un factor significativo.

Finalmente, la existencia de técnicos dedicados a actividades de I+D también influye positivamente en la adopción de métodos que contribuyen en la mejora de gestión de las relaciones externas, así como las financiaciones públicas por parte de la Administración del Estado y la Unión Europea.

\section{Conclusiones}

Como se demuestra con los resultados obtenidos del modelo, y a pesar de ser un tema relevante en la gestión empresarial de las empresas en general, y en particular de las innovaciones, el estudio de las IO ha recibido en general poca atención.

La falta de una definición ampliamente aceptada y las dificultades en la medición del concepto de IO ha retrasado sin duda la aparición de evidencia empírica respecto a este tipo de innovaciones. La base de datos utilizada en esta investigación (PITEC) permite solventar este problema, ya que aborda explícitamente la medición de las IO, utilizando para ello la definición proporcionada en el Manual de Oslo. La integración de esta definición en la encuesta constituye un avance notable en el seguimiento de las innovaciones no tecnológicas, en tanto que permite generar evidencia empírica comparable, sobre la base de una definición estandarizada, ampliamente aceptada y compartida a nivel internacional.

En un esfuerzo por avanzar en la comprensión de los factores que influyen sobre la adopción de IO, se estimó un modelo probit trivariante para identificar las variables determinantes en la adopción de los tres tipos distintos de innovación organizacional recogidos en PITEC.

Los resultados del análisis de los determinantes de la adopción de IO muestran los siguientes hallazgos. En primer lugar, la implementación del modelo probit trivariante, destaca la importancia de analizar la incorporación de IO como una acción que se lleva a cabo de manera interdependiente entre los tres tipos de innovación organizacional. Este hecho supone que el análisis de dichas innovaciones debe realizarse teniendo en cuenta lo que sucede con el resto de decisiones organizacionales.

Se puede afirmar que las IO están positivamente influidas por el tamaño, la introducción de TIC, la adquisición en maquinaria, software y hardware, la introducción de innovaciones en el mercado, el uso de protección, la solicitud de patentes (en menor medida), la cualificación de los recursos humanos disponibles, la necesidad de contar con consultores externos expertos y la necesidad de financiación pública de las empresas para hacer frente a los costes para la realización de actividades de innovación no tecnológica.

Los resultados muestran que aquellas variables que refuerzan la capacidad de aprendizaje de la organización influyen significativamente en la adopción de los tres tipos de IO. Este resultado sugiere que la interdependencia entre los distintos tipos 
de innovación organizacional responde a la existencia de elementos que realzan la introducción de innovaciones de forma transversal.

Este artículo constituye un punto de partida para continuar avanzando en la investigación de las IO. Las futuras líneas de investigación que se plantean pasan por un análisis temporal más amplio del efecto de las IO, así como un estudio más detallado de sus determinantes, que preste atención especial a la introducción de innovaciones en función de la clasificación sectorial (empresas manufactureras y de servicios) o de la capacidad tecnológica de la empresa (alta, media y baja capacidad tecnológica).

\section{Bibliografía}

Afcha Chávez, S. M. (2011). Behavioural additionality in the context of regional innovation policy in Spain. Innovation, 13(1), 95-110. doi:10.5172/ impp.2011.13.1.95

Albers, J. A., y Brewer, S. (2003). Knowledge management and the innovation process: The ecoinnovation model. Journal of Knowledge Management Practice, 4(3). Retrieved from http://www.tlainc.com/articl52.htm

Armbruster, H., Bikfalvi, A., Kinkel, S., y Lay, G. (2008). Organizational innovation: The challenge of measuring non-technical innovation in large-scale surveys. Technovation, 28(10), 644-657. doi:10.1016/j.technovation.2008.03.003

Balconi, M. (2002). Tacitness, codification of technological knowledge and the organisation of industry. Research Policy, 31(3), 357-379. doi:10.1016/ s0048-7333(01)00113-5

Barney, J. (1991). Firm Resources and Sustained Competitive Advantage. Journal of Management, 17(1), 99-120. doi:10.1177/014920639101700108

Birkinshaw, J., Hamel, G., y Mol, M. J. (2008). Management Innovation. Academy of Management Review, 33(4), 825-845. doi:10.5465/amr.2008.34421969

Brusoni, S., Marsili, O., y Salter, A. (2005). The role of codified sources of knowledge in innovation: Empirical evidence from Dutch manufacturing. Journal of Evolutionary Economics, 15(2), 211-231. doi:10.1007/s00191-005-0244-1

Burgelman, R., Maidique, M. A., y Wheelwright, S. C. (2005). Strategic management of technology and innovation. New York, NY: McGraw-Hill (5th edition). doi:10.1016/s0737-6782(97)90049-7

Caroli, E., y Van Reenen, J. (2001). Skill-Biased Organizational Change? Evidence from A Panel of British and French Establishments. The Quarterly Journal of Economics, 116(4), 1449-1492. doi:10.1162/003355301753265624

Chang, S., y Lee, M. (2008). The linkage between knowledge accumulation capability and organizational innovation. Journal of Knowledge Management, 12(1), 3-20. doi:10.1108/13673270810852359

Chen, Y. S., James Lin, M. J., y Chang, C. H. (2006). The Influence of Intellectual Capital on New Product Development Performance - The Manufacturing 
Companies of Taiwan as an Example. Total Quality Management \& Business Excellence, 17(10), 1323-1339. doi:10.1080/14783360601058979

Cohen, W. M., y Levinthal, D. A. (1990). Absorptive Capacity: A New Perspective on Learning and Innovation. Administrative Science Quarterly, 35(1), 128. doi: $10.2307 / 2393553$

Daft, R. L. (1978). A Dual-Core Model of Organizational Innovation. Academy of Management Journal, 21(2), 193-210. doi:10.2307/255754

Damanpour, F., y Evan, W. M. (1984). Organizational Innovation and Performance: The Problem of “Organizational Lag.” Administrative Science Quarterly, 29(3), 392. doi:10.2307/2393031

Damanpour, F., Walker, R. M., y Avellaneda, C. N. (2009). Combinative Effects of Innovation Types and Organizational Performance: A Longitudinal Study of Service Organizations. Journal of Management Studies, 46(4), 650-675. doi:10.1111/j.1467-6486.2008.00814.x

De Carolis, D. M., y Deeds, D. L. (1999). The Impact of Stocks and Flows of Organizational Knowledge on Firm Performance: An Empirical Investigation of the Biotechnology Industry. Strategic Management Journal, 20, 953-968. https://doi. org/10.1002/(sici)1097-0266(199910)20:10<953::aid-smj59>3.0.co;2-3

De Carolis, D. M. (2003). Competencies and Imitability in the Pharmaceutical Industry: An Analysis of Their Relationship with Firm Performance. Journal of Management, 29(1), 27-50. doi:10.1177/014920630302900103

De Propris, L. D. (2000). Innovation and Inter-Firm Co-Operation: The Case Of The West Midlands. Economics of Innovation and New Technology, 9(5), 421-446. doi:10.1080/10438590000000017

Dougherty, D. (1992). Interpretive Barriers to Successful Product Innovation in Large Firms. Organization Science, 3(2), 179-202. doi:10.1287/orsc.3.2.179

Edmondson, A. C., Winslow, A. B., Bohmer, R. M. J., y Pisano, G. P. (2003). Learning How and Learning What: Effects of Tacit and Codified Knowledge on Performance Improvement Following Technology Adoption. Decision Sciences, 34(2), 197-224. doi:10.1111/1540-5915.02316

Eisenhardt, K. M., y Martin, J. A. (2000). Dynamic Capabilities: What are They? Strategic Management Journal, 21, 1105-1121. https://doi.org/10.1002/ 1097-0266(200010/11)21:10/11<1105:aid-smj133>3.0.co;2-e

EUROSTAT (2005). Oslo Manual. The Measurement of Scientific and Technological Activities. doi:10.1787/9789264013100-en

Evans, P., Pucik, V., y Barsoux, J. L. (2002). The global challenge: Frameworks for international human resource management. McGraw-Hill. https://doi. org/10.1177/030630700202700406

Fariñas, J., y Lopez, A. (2011). Medición de las innovaciones organizativas y efectos sobre la productividad: Evidencias sobre la empresa española. Papeles de Economía Española, 127, pp. 120-139. http://www.funcas.es/publicaciones_new/ publicaciones.aspx 
FECYT (2012). La base de datos Panel de Innovación Tecnológica (PITEC). FECYT. Madrid. Disponible en: http://icono.fecyt.es/05\%29Publi/AA\%29panel/basedatosPITEC_Mayo_2011_esp.pdf

Fierro, E., Mercado, P., y Cernas, D. A. (2013). El efecto de la cultura centrada en el conocimiento y la interacción social en la innovación organizativa. El efecto mediador de la gestión del conocimiento. Esic Market Economics and Business Journal. Vol. 44, n. ${ }^{\circ}$ 2, mayo-agosto 2013, 87-108. DOI: 10.7200/ esicm.145.0442.2e

Geroski, P. (1995). Innovation and Competitive Advantage. OECD Economics Department Working Papers. Paris, Francia. https://doi.org/10.1787/ 344434438114

Goldman, S. L., Nagel, R. N., Preiss, K., y Warnecke, H. J. (1996). Mit dem Kunden Gemeinsam Agile Geschäftsstrukturen Erarbeiten. In: Agil im Wettbewerb. Springer, Berlin, Heidelberg. doi.org/10.1007/978-3-642-61101-8_8

Grant, R. (1999). The Resource-Based Theory of Competitive Advantage: Implications for Strategy Formulation. Knowledge and Strategy, 3-23. doi:10.1016/ b978-0-7506-7088-3.50004-8

Grant, R. M. (1996). Toward a knowledge-based theory of the firm. Strategic Management Journal, 17(S2), 109-122. doi:10.1002/smj.4250171110

Greenan, N. (2003). Organisational change, technology, employment and skills: an empirical study of French manufacturing. Cambridge Journal of Economics, 27(2), 287-316. doi:10.1093/cje/27.2.287

Gu, W., y Gera, S. (2004). The Effect of Organizational Innovation and Information Technology on Firm Performance. SSRN Electronic Journal. doi:10.2139/ ssrn.1404689

Guan, J. C., Yam, R. C. M., Tang, E. P. Y., y Lau, A. K. W. (2009). Innovation strategy and performance during economic transition: Evidences in Beijing, China. Research Policy, 38(5), 802-812. doi:10.1016/j.respol.2008.12.009

Hadjimanolis, A. (2003). The Barriers Approach to Innovation. The International Handbook on Innovation, 559-573. doi:10.1016/b978-008044198-6/50038-3

Hammer, M., y Champy, J. (1993). Reengineering the corporation: A manifesto for business revolution. Business Horizons, 36(5), 90-91. doi:10.1016/ s0007-6813(05)80064-3

Henderson, R. (1993). Underinvestment and Incompetence as Responses to Radical Innovation: Evidence from the Photolithographic Alignment Equipment Industry. The RAND Journal of Economics, 24(2), 248. doi:10.2307/2555761

Henderson, R., y Cockburn, I. (1994). Measuring Competence? Exploring Firm Effects in Pharmaceutical Research. Strategic Management Journal, 15(S1), 63-84. doi:10.1002/smj.4250150906

Holden, N., y Glisby, M. (2010). Creating Knowledge Advantage. The Tacit Dimension of International Competition and Cooperation.

Hwang, H. G., Chang, I. C., Chen, F. J., y Wu, S. Y. (2008). Investigation of the application of KMS for diseases classifications: A study in a Taiwanese hospital. Expert Systems with Applications, 34(1), 725-733. doi:10.1016/j.eswa.2006.10.018 
Kleinschmidt, E. J., de Brentani, U., y Salomo, S. (2007). Performance of Global New Product Development Programs: A Resource-Based View. Journal of Product Innovation Management, 24(5), 419-441. doi:10.1111/j.1540-5885.2007.00261.x

Kusunoki, K., Nonaka, I., y Nagata, A. (1998). Organizational Capabilities in Product Development of Japanese Firms: A Conceptual Framework and Empirical Findings. Organization Science, 9(6), 699-718. doi:10.1287/orsc.9.6.699

Lam, A. (2006). Organizational Innovation. Oxford Handbooks Online. doi:10.1093/ oxfordhb/9780199286805.003.0005

Leiponen, A., y Helfat, C. E. (2009). Innovation objectives, knowledge sources, and the benefits of breadth. Strategic Management Journal, 31(2), 224-236. doi:10.1002/smj.807

Leonard-Barton, D. (1992). Core capabilities and core rigidities: A paradox in managing new product development. Strategic Management Journal, 13(S1), 111-125. doi:10.1002/smj.4250131009

Liao, S. H., y Wu, C. (2010). System perspective of knowledge management, organizational learning, and organizational innovation. Expert Systems with Applications, 37(2), 1096-1103. doi:10.1016/j.eswa.2009.06.109

Liebeskind, J. P. (1996). Knowledge, strategy, and the theory of the firm. Strategic Management Journal, 17(S2), 93-107. doi:10.1002/smj.4250171109

McEvily, S. K., y Chakravarthy, B. (2002). The persistence of knowledge-based advantage: an empirical test for product performance and technological knowledge. Strategic Management Journal, 23(4), 285-305. doi:10.1002/smj.223

Miller, D. J., Fern, M. J., y Cardinal, L. B. (2007). The Use of Knowledge for Technological Innovation Within Diversified Firms. Academy of Management Journal, 50(2), 307-325. doi:10.5465/amj.2007.24634437

Mohnen, P., y Röller, L. H. (2005). Complementarities in innovation policy, European Economic Review. Volume 49, Issue 6, August 2005, 1431-1450. doi. org/10.1016/j.euroecorev.2003.12.003

Nonaka, I. (1994). A Dynamic Theory of Organizational Knowledge Creation. Organization Science, 5(1), 14-37. doi:10.1287/orsc.5.1.14

Nonaka, I., y Toyama, R. (2005). The theory of the knowledge-creating firm: subjectivity, objectivity and synthesis. Industrial and Corporate Change, 14(3), 419-436. doi:10.1093/icc/dth058

Organización para la Cooperación y el Desarrollo Económicos (OCDE) (2015). Frascati Manual 2015: Guidelines for Collecting and Reporting Data on Research and Experimental Development, The Measurement of Scientific, Technological and Innovation Activities, OECD Publishing, Paris, https://doi. org/10.1787/9789264239012-en

Peteraf, M. A. (1993). The cornerstones of competitive advantage: A resourcebased view. Strategic Management Journal, 14(3), 179-191. doi:10.1002/smj. 4250140303

Piva, M., Santarelli, E., y Vivarelli, M. (2005). Technological and organizational changes as determinants of the skill bias: evidence from the Italian machinery industry. Managerial and Decision Economics, 27(1), 63-73. doi:10.1002/mde.1246 
Polder, M., Leeuwen, G. van, Mohnen, P., y Raymond, W. (2010). Product, Process and Organizational Innovation: Drivers, Complementarity and Productivity Effects. SSRN Electronic Journal. doi:10.2139/ssrn.1626805

Prajogo, D. I., y Ahmed, P. K. (2006). Relationships between innovation stimulus, innovation capacity, and innovation performance. R\&D Management, 36(5), 499-515. doi:10.1111/j.1467-9310.2006.00450.x

Roberts, P. W., y Amit, R. (2003). The Dynamics of Innovative Activity and Competitive Advantage: The Case of Australian Retail Banking, 1981 to 1995. Organization Science, 14(2), 107-122. doi:10.1287/orsc.14.2.107.14990

Schulz, M., y Jobe, L. A. (2001). Codification and tacitness as knowledge management strategies: an empirical exploration. The Journal of High Technology Management Research, 12(1), 139-165. doi:10.1016/s1047-8310(00)00043-2

Sher, P. J., y Yang, P. Y. (2005). The Effects of Innovative Capabilities and R\&D Clustering on Firm Performance: The Evidence of Taiwan's Semiconductor Industry. Technovation 25, 33-43. doi.org/10.1016/S0166-4972(03)00068-3

Souitaris, V. (2002). Firm-specific competencies determining technological innovation: A survey in Greece. $R$ y D Management, 32(1), 61-77. doi:10.1111/ 1467-9310.00239

Spender, J. C., y Grant, R. M. (1996). Knowledge and the firm: Overview. Strategic Management Journal, 17(S2), 5-9. doi:10.1002/smj.4250171103

Subramaniam, M., y Youndt, M. A. (2005). The Influence of Intellectual Capital on the Types of Innovative Capabilities. Academy of Management Journal, 48, 450463. http://dx.doi.org/10.5465/AMJ.2005.17407911

Teece, D. J., Pisano, G., y Shuen, A. (1997). Dynamic Capabilities and Strategic Management. Strategic Management Journal, 18, 509-533. https://doi. org/10.1002/(sici)1097-0266(199708)18:7<509::aid-smj882>3.0.co;2-z

Tourigny, D., y Le, C. D. (2004). Impediments to innovation faced by Canadian manufacturing firms. Economics of Innovation and New Technology, 13(3), 217-250. doi:10.1080/10438590410001628387

Varela, J. A., y Méndez, J. N. (2017). Relación entre factores administrativos e innovación. Revista EAN, 83, pp. 31-50. https://doi.org/10.21158/01208160. n83.2017.1826

Womack, J., Jones, D., y Roos, D. (1992). The machine that changed the world. Business Horizons, 35(3), 81-82. doi:10.1016/0007-6813(92)90074-j

Yamakawa, P., y Ostos, J. (2012). Relación entre innovación organizacional y desempeño organizacional. Revista Universidad \& Empresa. 21, 93-115

Yang, H. L., y Hsiao, S. L. (2009). Mechanisms of developing innovative IT-enabled services: A case study of Taiwanese healthcare service. Technovation, 29(5), 327-337. doi:10.1016/j.technovation.2009.01.006 


\section{Datos de la autora}

Nombre: M. Piedad Fernández Perea

Cargo: Doctorando. Profesora de Secundaria. Comunidad de Madrid

Escuela/Facultad: Escuela Internacional de Doctorado (EIDUNED). Área de Economía y Empresa

Universidad: Universidad Nacional de Educación a Distancia (UNED)

Dirección: Calle de Bravo Murillo, $38-3 .^{\text {a }}$, 28015, Madrid

Teléfono: 913987494

Correo electrónico: piedad.fernandez.perea@gmail.com 